\title{
Agregação de comportamentos de risco à saúde de escolares da rede pública de Jequié, Bahia, Brasil
}

\section{Aggregation of health behaviors risk of schools of the public network of Jequié, Bahia, Brazil}

\section{AUTORES}

Hector Luiz Rodrigues Munaro ${ }^{1}$ (D)

Diego Augusto Santos Silva ${ }^{1}$ (D)

Adair da Silva Lopes ${ }^{1}$ (ID

1 Universidade Federal de Santa Catarina - UFSC, Centro de Desportos, Florianópolis, Santa Catarina, Brasil.

\section{CONTATO}

Hector Luiz Rodrigues Munaro

blrmunaro@uesb.edu.br

Universidade Estadual do Sudoeste da Bahia

Av. José Moreira Sobrinho - SN. UESB, Campus de Jequié, Departamento de Saúde I, Bairro Jequiezinho, Jequié, Bahia, Brasil. CEP: 45206-180.

DOI

$10.12820 /$ rbafs. $23 \mathrm{e} 0016$

\section{(c) $(1) \Theta$ BY NC}

Copyright: This is an open-access article distributed under the terms of the Creative Commons Attribution License ${ }^{\circledast}$, which permits unrestricted use, distribution, and reproduction in any medium, provided that the original author and source are credited.

\begin{abstract}
RESUMO
O objetivo deste estudo foi estimar as prevalências de comportamentos de risco à saúde, de forma isolada e agregada, e analisar fatores associados. Tratou-se de estudo epidemiológico transversal, com amostra representativa de escolares de 14 a 20 anos de idade $(n=1.170)$ do ensino médio da rede pública, Jequié, Bahia, Brasil. Os comportamentos de risco observados foram: níveis insuficientes de atividade física, comportamento sedentário, consumo inadequado de frutas e verduras e consumo de bebidas alcoólicas e de tabaco. Os possíveis fatores associados foram: sexo, idade, estado civil, ocupação, escolaridade da mãe e renda familiar. A prevalência de nível insuficiente de atividade física foi mais elevada no sexo feminino $(87,6 \%$ vs. $73,0 \%$; $p=0,001)$, enquanto que o consumo de bebidas alcoólicas $(28,2 \%$ vs. $20,6 \% ; \mathrm{p}=0,003)$ e de tabaco $(8,7 \%$ vs. $3,8 \% ; \mathrm{p}=0,001)$ foi superior no masculino. Para o sexo feminino houve 6,34 vezes maior a prevalência observada pela esperada (PO/ $\mathrm{PE}$ ), entre: níveis insuficientes de atividade física, comportamento sedentário e consumo de bebidas alcoólicas e de tabaco A prevalência de três ou mais comportamentos de risco foi de 7,9\% (IC95\%: 6,4-9,5) e os escolares que pertenciam às famílias com renda inferior a dois salários mínimos tiveram menor probabilidade de exposição ( $\mathrm{RP}=0,60$; IC95\%: 0,39-0,93). Os resultados indicaram elevada prevalência de insuficientemente ativos, de consumo inadequado de frutas e verduras e agregação de múltiplos comportamentos de risco para o sexo feminino.
\end{abstract}

Palavras-chave: Fatores de risco; Adolescente; Estilo de vida.

ABSTRACT

The aim of this study to estimate the health behaviors risks prevalence in isolated and aggregated way, and analyze associated factors. This was a cross-sectional epidemiological study, with a representative sample of public high school students, from 14 to 20 years of age $(n=1,170)$ from Jequie, Babia, Brazil. The observed behaviors risks were: insufficient levels of physical activity, sedentary behavior, inadequate fruits and vegetable consumption, and alcoholic beverages and tobacco consumption. Possible associated factors were: gender, age, marital status, occupation, mother's education, and family income. The insufficient physical activity prevalence was higher in females ( $87.6 \%$ ws. $73.0 \%, p=0.001)$, while alcohol consumption $(28.2 \%$ vs. $20.6 \% ; p=0.003)$ and tobacco (8.7\% vs. 3.8\%, $p=0.001)$ was higher in males. For female sex, there was a 6.34-fold higher observed for the expected prevalence $(O P / E P)$, between insufficient physical activity levels, sedentary behavior, and alcoholic beverages and tobacco consumption. The three or more risk behaviors prevalence was 7.9\% (95\%CI: 6.4-9.5) and schoolchildren belonging to families with incomes less than two minimum wages had a lower probability of exposure ( $P R=0.60$; $95 \% C I: 0.39-0.93)$. The results indicated a high prevalence of insufficiently active, inadequate fruits and vegetable consumption and the aggregation of multiple behaviors risk for the female sex.

Keywords: Risk factors; Adolescent; Life style.

\section{Introdução}

A exposição a alguns comportamentos de risco à saúde, isolados ou agregados, como níveis insuficientes de atividade física, comportamento sedentário excessivo, consumo inadequado de frutas e verduras, uso de bebidas alcoólicas e de tabaco, na adolescência, pode acarretar em aumento no risco de desenvolvimento de doenças cardiovasculares, sobrepeso/obesidade e al- guns tipos de cânceres, além de aumentarem os riscos de mortalidade precoce na idade adulta ${ }^{1,2}$.

Estudos prévios que investigaram a agregação de comportamentos de risco à saúde na população adulta utilizaram quatro comportamentos de risco de forma combinada $^{3,4}$. Estudo realizado no Brasil (Florianópolis, Santa Catarina) identificou que 7,2\% da amostra estava exposta a três comportamentos de risco agregados (dieta 
inadequada, tabagismo e níveis insuficientes de atividade física $)^{3}$. Estudo com adultos da Inglaterra ${ }^{4}$ identificou que $23 \%$ estavam expostos a pelo menos três comportamentos de risco (dieta inadequada, uso de bebidas alcoólicas e níveis insuficientes de atividade física).

Em adolescentes, grande parte dos estudos investigou a agregação de fatores de risco cardiovasculares (excesso de peso corporal, pressão arterial elevada e hiperglicemia) $)^{5,6}$. Estudos realizados com adolescentes brasileiros $^{7,8}$, e que analisaram a agregação de quatro comportamentos de risco e seus respectivos fatores associados, demonstraram resultados distintos: adolescentes de Pelotas (Rio Grande do Sul) - a exposição a três ou mais comportamentos de risco à saúde foi mais elevada no sexo feminino, nos maiores de 15 anos e de baixa renda familiar ${ }^{7}$; escolares de 14 a 20 anos de idade, da cidade de Caruaru (Pernambuco) - maior exposição nos casos de maior renda familiar, que estudavam no turno matutino de ensino e que não participavam das aulas de Educação Física na escola ${ }^{8}$.

Revisão sistemática prévia ${ }^{9}$, com o objetivo de identificar os estudos de agregação de comportamentos de risco entre adolescentes, em especial os níveis insuficientes de atividade física, o comportamento sedentário excessivo e a dieta inadequada, identificou que dos 18 estudos selecionados, seis incluíram, também, o consumo de bebidas alcoólicas e de tabaco. Em nove estudos foram identificadas associações com variáveis socioeconômicas, sendo a maior parte relacionada ao nível de instrução dos pais e renda familiar.

Desta forma, pela escassez de levantamentos epidemiológicos com escolares na Bahia, o objetivo do estudo foi estimar as prevalências dos comportamentos de riscos à saúde (níveis insuficientes de atividade física, comportamento sedentário, consumo inadequado de frutas e verduras e uso de bebidas alcoólicas e de tabaco), a agregação destes comportamentos de risco e os fatores sociodemográficos (sexo, faixa etária, ocupação, renda familiar e escolaridade da mãe) associados à presença simultânea de comportamentos de risco em escolares do ensino médio da rede pública da cidade de Jequié, Bahia, Brasil.

\section{Metodos}

Estudo epidemiológico transversal integrante de um monitoramento de comportamentos de risco à saúde em escolares do ensino médio da rede estadual de ensino na cidade de Jequié, Bahia, Brasil. O município de Jequié se localiza na região Sudoeste do Estado, distante, aproximadamente, $370 \mathrm{~km}$ de Salvador, com população estimada em 151.895 habitantes e Índice de Desenvolvimento Humano (IDH) de $0,694^{10}$.

A população deste estudo compreendeu 3.040 escolares, de 98 turmas de todas as 12 escolas públicas estaduais urbanas do município, devidamente matriculados no ensino médio nos turnos matutino e vespertino, em 2015. Esta população representava 45,9\% dos escolares matriculados no município em comparação à oferta em estabelecimentos privados ou municipais ${ }^{10}$. A seleção da amostra foi por conglomerados em dois estágios ${ }^{11}$.

No primeiro estágio, a unidade amostral foi a escola. Foram selecionados aqueles que ofereciam ensino médio no tuno diurno (matutino e vespertino) e estavam localizadas na área urbana $(\mathrm{n}=12)$. Foram excluídas as escolas das áreas rurais $(\mathrm{n}=3)$ e o Colégio da Polícia Militar, onde há sistema de seleção para vagas e o modelo de ensino difere dos demais.

No segundo estágio, a unidade amostral foram as turmas de ensino médio, selecionadas de maneira proporcional ao número de séries em cada escola, pois haviam escolas de maior porte, com número de classes por séries distintos. A amostra foi composta por 48 turmas, sendo que todas as escolas tiveram pelo menos uma turma de cada série, sendo mantida a proporcionalidade de representação de cada escola.

O parâmetro para a determinação do tamanho da amostra foi a prevalência estimada do fenômeno igual a 50\%, com intervalo de confiança de 95\%, erro máximo aceitável de três pontos percentuais, efeito do delineamento de 1,5 , e $15 \%$ para os casos de perdas ou recusas. Assim, chegou-se a uma amostra mínima de 1.388 escolares.

A coleta de dados ocorreu nos meses de julho e agosto de 2015, sendo utilizado o questionário COM$\mathrm{PAC}^{12}$, aplicado em sala de aula por pesquisadores previamente treinados, com duração média de 28 minutos para o seu preenchimento pelos escolares. Este instrumento apresentou bons índices de reprodutibilidade $(0,51$ a 0,97$)$. As variáveis sociodemográficas foram: sexo (masculino ou feminino), faixa etárias (< 16 anos ou $\geq 16$ anos), estado civil (solteiro(a) ou casado(a)/ outro), ocupação (não trabalha ou trabalha), renda familiar (< dois salários mínimos ou $\geq$ dois salários mínimos)e escolaridade da mãe (< oito anos de estudo ou $\geq$ oito anos de estudos).

Os comportamentos de risco à saúde foram operacionalizados na forma que segue:

Insuficientemente Ativos (IA): foram utilizadas 
duas questões sobre a frequência da atividade física, sendo: "Durante uma semana normal (típica), em quantos dias você pratica atividades físicas moderadas a vigorosas (atividade física no lazer, no trabalho e no deslocamento)?"12; e outra questão sobre a duração: "Durante uma semana normal (típica), quanto tempo você pratica atividades físicas moderadas a vigorosas (atividade física no lazer, no trabalho e no deslocamento)?"12. Após combinação das duas variáveis, foram considerados insuficientemente ativos aqueles que não acumulavam o mínimo recomendado de, pelo menos, cinco dias na semana e 60 minutos por $\mathrm{dia}^{13}$;

Comportamento Sedentário (CS): foram autorreferidas pelos escolares em duas questões, sendo a primeira sobre televisão: "Quantas horas por dia você assiste televisão?" ${ }^{2}$, e a segunda: "Quantas horas por dia você usa computador e/ou joga videogame?"12. Foi considerado excessivo tempos $\geq 2$ horas/dia no somatório dos dois comportamentos;

Frutas e Verduras (FV): foi utilizado como critério o consumo de uma porção diária de frutas e verduras durante uma semana típica ${ }^{12}$; após a combinação das duas variáveis, foi considerado como consumo inadequado $<5$ dias/semana ${ }^{15}$;

Bebidas Alcoólicas (BA): foram utilizadas a duas perguntas, sendo: "Durante uma semana normal (típica), em quantos dias você consome bebidas alcoólicas?", e a outra: "Durante uma semana normal (típica), nos dias em que você consome bebidas alcoólicas, quantas doses você consome por dia?"12. Após a combinação das variáveis, como critério, o consumo de álcool foi determinado independentemente do número de doses e foram considerados expostos aqueles que responderam acima de uma dose por dia e pelo menos um dia na semana;

Tabaco (T): foi utilizada uma pergunta: "Com relação ao fumo, qual a sua situação?"12, utilizando como critério o consumo independentemente do número de cigarros, e considerados expostos aqueles que responderam que consumiam mais de um cigarro por dia.

O teste do Qui-quadrado foi utilizado para comparar as proporções das variáveis sociodemográficas e do estilo de vida entre os sexos.

A agregação foi considerada quando a combinação da prevalência observada ( $\mathrm{PO}$ ) dos comportamentos de risco superou a prevalência esperada $(\mathrm{PE})^{3-12}$. A prevalência esperada de cada combinação de comportamentos de risco à saúde foi obtida por meio da multiplicação da probabilidade individual da ocorrência de cada comportamento com base na ocorrência observada na amostra. Por exemplo, considere as prevalências observadas para os cinco comportamentos de risco: insuficientemente ativos $=81,5 \%$; comportamento sedentário = 11,4\%; frutas e verduras = 24,4\%; bebidas alcoólicas $=23,8 \%$; e, tabaco $=5,9 \%$. A agregação seria: $0,815 \times 0,114 \times 0,244 \times 0,238 \times 0,059=0,003(0,3 \%)$. Entretanto, a prevalência esperada para os mesmos comportamentos agregados foi de $0,2 \%$ e, neste caso, a $\mathrm{PO} / \mathrm{PE}$ foi de 1,5 . A razão da prevalência observada pela esperada (PO/PE) superior a 1 significa a agregação entre os comportamentos de risco à saúde.

A regressão de Poisson, com variância robusta ${ }^{14}$, foi realizada com o intuito de analisar a relação entre a variável dependente (exposição simultânea a três ou mais comportamentos de risco) e as variáveis sociodemográficas (sexo, faixa etária, estado civil, ocupação, renda familiar e escolaridade da mãe) com Intervalo de Confiança (IC) de 95\%, incluídas no ajustamento todos os potenciais fatores de confusão (variáveis sociodemográficas).

O estudo foi aprovado pelo Comitê de Ética em Pesquisa com Seres Humanos da Universidade Estadual do Sudoeste da Bahia (83.957/14). Os escolares que participaram do estudo foram autorizados pelos pais e aqueles com 18 anos ou mais assinaram o próprio Termo de Consentimento Livre e Esclarecido.

\section{Resultados}

Inicialmente, a amostra foi composta por 1.388 escolares e $16,8 \%(n=218)$ foram recusas e perdas. Sendo assim, a amostra final foi composta por 1.170 escolares de ambos os sexos $(57,9 \% ; n=678)$. Em relação às variáveis sociodemográficas, o sexo feminino apresentou maiores proporções entre aqueles que não trabalhavam ( $88,6 \%$ vs. 71,$3 ; p=0,000)$, cuja escolaridade das mães era inferior a oito anos de estudo (41,2\% vs. 33,7\%; $\mathrm{p}=$ $0,012)$ e renda familiar inferior a dois salários mínimos $(76,7 \%$ vs. $63,8 \% ; \mathrm{p}=0,000)$.

Os comportamentos de risco mais prevalentes foram: níveis insuficientes de atividade física e consumo inadequado de frutas e verduras. A prevalência de insuficientemente ativos foi mais elevada no sexo feminino ( $87,6 \%$ vs. $73 \% ; p=0,001)$, enquanto que consumo de bebidas alcoólicas $(28,2 \%$ vs. 20,6\%; $\mathrm{p}=0,003)$ e de tabaco $(8,7 \%$ vs. $3,8 \% ; \mathrm{p}=0,001)$ foi mais elevado no sexo masculino - Tabela 1. A prevalência estimada da exposição simultânea a três ou mais comportamentos de risco à saúde foi de 7,9\% (IC95\%: 6,4-9,5). 
Tabela 1 - Características descritivas da amostra segundo as variáveis sociodemográficas e do estilo de vida. Jequié, Bahia, Brasil, 2015.

\begin{tabular}{|c|c|c|c|c|c|}
\hline \multirow{2}{*}{ Variáveis } & \multicolumn{2}{|c|}{ Masculino } & \multicolumn{2}{|c|}{ Feminino } & \multirow{2}{*}{$\frac{\mathrm{X}^{2}}{\mathrm{P}}$} \\
\hline & $\%$ & $\mathrm{n}$ & $\%$ & $\mathrm{n}$ & \\
\hline \multicolumn{6}{|l|}{ Sociodemográficas } \\
\hline \multicolumn{6}{|l|}{ Idade (anos) } \\
\hline$<16$ & 48,8 & 240 & 54,4 & 369 & $0,065^{*}$ \\
\hline$\geq 16$ & 51,2 & 252 & 45,6 & 309 & \\
\hline \multicolumn{6}{|l|}{ Série (ano) } \\
\hline $1^{\mathrm{a}}$ & 42,5 & 209 & 41,2 & 279 & $0,282^{*}$ \\
\hline $2^{\mathrm{a}}$ & 32,5 & 160 & 29,8 & 202 & \\
\hline $3^{\mathrm{a}}$ & 25,0 & 123 & 29,1 & 197 & \\
\hline \multicolumn{6}{|l|}{ Estado civil } \\
\hline Solteiro(a) & 90,0 & 443 & 87,3 & 592 & $0,178^{*}$ \\
\hline Casado(a)/outro & 10,0 & 49 & 12,7 & 86 & \\
\hline \multicolumn{6}{|l|}{ Ocupação } \\
\hline Não trabalha & 71,3 & 351 & 88,6 & 601 & $0,000^{*}$ \\
\hline Trabalha & 28,7 & 141 & 11,4 & 77 & \\
\hline \multicolumn{6}{|l|}{ Escolaridade da mãe (anos de estudo) } \\
\hline$<8$ anos & 33,7 & 166 & 41,2 & 279 & $0,012^{*}$ \\
\hline$\geq 8$ anos & 66,3 & 326 & 58,8 & 379 & \\
\hline \multicolumn{6}{|l|}{ Renda familiar mensal (mínimos) } \\
\hline$<2$ Salários & 63,8 & 314 & 76,7 & 520 & $0,000^{*}$ \\
\hline$\geq 2$ Salários & 36,2 & 178 & 23,3 & 158 & \\
\hline \multicolumn{6}{|l|}{ Comportamentos de risco (isolados) } \\
\hline Níveis insuficientes de atividade física & 73,0 & 359 & 87,6 & 594 & $0,000^{*}$ \\
\hline Comportamento sedentário excessivo & 11,1 & 58 & 11,8 & 75 & $0,769^{*}$ \\
\hline $\begin{array}{l}\text { Consumo inadequado de frutas e } \\
\text { verduras }\end{array}$ & 19,9 & 98 & 27,6 & 187 & $0,003^{*}$ \\
\hline Consumo de bebidas alcoólicas & 28,2 & 138 & 20,6 & 139 & $0,004^{*}$ \\
\hline Consumo de tabaco & 8,7 & 43 & 3,8 & 26 & $0,001^{*}$ \\
\hline \multicolumn{6}{|c|}{ Número de comportamentos de risco (combinados) } \\
\hline 0 & 11,4 & 56 & 3,8 & 26 & $0,000^{* *}$ \\
\hline 1 & 47,6 & 234 & 53,4 & 362 & $0,049^{* *}$ \\
\hline 2 & 30,7 & 151 & 31,1 & 211 & $0,926^{* *}$ \\
\hline 3 & 7,9 & 39 & 6,2 & 42 & $0,249^{* *}$ \\
\hline 4 & 1,4 & 7 & 1,2 & 8 & $0,716^{* *}$ \\
\hline 5 & 0,2 & 1 & 0 & 0 & $0,240^{* *}$ \\
\hline
\end{tabular}

${ }^{*} \mathrm{X}^{2}=$ Teste qui-quadrado para heterogeneidade; ${ }^{* *} \mathrm{X}^{2}=$ Teste qui-quadrado para tendência linear.

$\mathrm{Na}$ Tabela 2 são apresentadas as informações sobre a agregação de comportamentos de risco à saúde. Foram identificados 32 padrões de agregação e, em 22 deles, a prevalência observada foi maior que a esperada. Os insuficientemente ativos estiveram agregados aos outros comportamentos de risco em oito padrões para ambos os sexos.
No sexo masculino, a agregação que incluiu bebidas alcoólicas e tabaco foi 5,87 vezes maior a prevalência observada comparada a esperada (Tabela 2). No sexo feminino, o padrão de agregação que incluiu níveis insuficientes de atividade física, o comportamento sedentário, o consumo de bebidas alcoólicas e de tabaco foi 6,34 vezes maior a prevalência observada comparada a esperada.

$\mathrm{Na}$ análise bruta, observou-se que apenas aqueles com renda familiar inferior a dois salários mínimos apresentaram associação ao desfecho $\left(\mathrm{RP}_{\text {bruta }}=0,58\right.$; IC95\%: 0,39-0,87), mantendo-se associada após a análise ajustada pelos fatores de confusão $\left(\mathrm{RP}_{\text {ajustada }}=0,60\right.$; IC95\%: 0,38-0,93). As demais variáveis não estiveram associadas ao desfecho, tanto na análise bruta como na ajustada (Tabela 3).

Tabela 3 - Análise de regressão bruta e ajustada para associação entre variáveis sociodemográficas e a presença de três ou mais comportamentos de risco à saúde. Jequié, Bahia, Brasil, 2015.

\begin{tabular}{|c|c|c|c|c|c|}
\hline Variáveis & $\%$ & $\begin{array}{l}\text { RP bruta } \\
\text { (IC95\%) }\end{array}$ & $\mathrm{p}$ & $\begin{array}{l}\text { RP ajustada } \\
\text { (IC95\%) }\end{array}$ & $\mathrm{p}$ \\
\hline \multicolumn{6}{|l|}{$\overline{\text { Sexo }}$} \\
\hline Feminino & 4,2 & $0,84(0,56-1,25)$ & 0,381 & $0,91(0,60-1,40)$ & 0,676 \\
\hline Masculino & 3,7 & 1 & & 1 & \\
\hline \multicolumn{6}{|l|}{ Idade (anos) } \\
\hline$<16$ & 3,9 & $0,90(0,60-1,34)$ & 0,603 & $0,84(53-1,33)$ & 0,462 \\
\hline$\geq 16$ & 4,0 & 1 & & 1 & \\
\hline \multicolumn{6}{|l|}{ Série (ano) } \\
\hline $1^{\mathrm{a}}$ & 3,3 & $1,04(0,63-1,71)$ & 0,879 & $1,07(0,62-1,87)$ & 0,799 \\
\hline $2^{\mathrm{a}}$ & 2,3 & $1,11(0,66-1,88)$ & 0,683 & $1,14(0,67-1,93)$ & 0,615 \\
\hline $3^{\mathrm{a}}$ & 2,0 & 1 & & 1 & \\
\hline \multicolumn{6}{|l|}{ Estado civil } \\
\hline Solteiro(a) & 6,7 & $0,75(0,43-1,32)$ & 0,322 & $0,79(0,45-1,40)$ & 0,420 \\
\hline $\begin{array}{l}\text { Casado(a)/ } \\
\text { outro }\end{array}$ & 1,2 & 1 & & 1 & \\
\hline \multicolumn{6}{|l|}{ Ocupação } \\
\hline Não trabalha & 6,3 & $0,92(0,56-1,50)$ & 0,732 & $0,99(0,60-1,64)$ & 0,975 \\
\hline Trabalha & 1,3 & 1 & & 1 & \\
\hline \multicolumn{6}{|c|}{ Escolaridade da mãe (anos de estudo) } \\
\hline$<8$ anos & 2,7 & $0,84(0,55-1,28)$ & 0,426 & $0,93(0,60-1,43)$ & 0,732 \\
\hline$\geq 8$ anos & 5,2 & 1 & & 1 & \\
\hline \multicolumn{6}{|c|}{ Renda familiar mensal (salários mínimos) } \\
\hline$<2$ Salários & 4,7 & $0,58(0,39-0,87)$ & $0,009^{*}$ & $0,60(0,38-0,93)$ & $0,022^{*}$ \\
\hline$\geq 2$ Salários & 3,2 & 1 & & 1 & \\
\hline
\end{tabular}

$\mathrm{RP}=$ Razão de prevalência; $\mathrm{IC}=$ Intervalo de confiança de 95\%; * $\mathrm{p}<0,05$. 
Tabela 2 - Prevalências e agregação de cinco comportamentos de risco à saúde estratificadas por sexo. Jequié, Bahia, Brasil, 2015.

\begin{tabular}{|c|c|c|c|c|c|c|c|c|c|c|c|}
\hline \multirow{2}{*}{$\mathrm{n}$} & \multirow{2}{*}{ IA } & \multirow{2}{*}{$\mathrm{CS}$} & \multirow{2}{*}{$\mathrm{FV}$} & \multirow{2}{*}{$\mathrm{BA}$} & \multirow{2}{*}{$\mathrm{T}$} & \multicolumn{3}{|c|}{ Masculino } & \multicolumn{3}{|c|}{ Feminino } \\
\hline & & & & & & $\mathrm{O}(\%)$ & $\mathrm{E}(\%)$ & O/E (IC95\%) & $\mathrm{O}(\%)$ & $\mathrm{E}(\%)$ & $\mathrm{O} / \mathrm{E}(\mathrm{IC} 95 \%)$ \\
\hline 0 & - & - & - & - & - & 11,4 & 9,1 & $1,25(1,02-1,48)^{*}$ & 3,8 & 5,2 & $0,73(0,43-1,02)$ \\
\hline 1 & + & - & - & - & - & 36,6 & 24,6 & $1,49(1,37-1,61)^{*}$ & 46,5 & 37,0 & $1,26(1,14-1,37)^{*}$ \\
\hline 1 & - & + & - & - & - & 1,0 & 1,2 & $0,84(0,53-1,13)$ & 0 & 0,7 & 0 \\
\hline 1 & - & - & + & - & - & 4,7 & 6,5 & $0,72(0,47-0,97)$ & 4,4 & 3,2 & $1,38(1,07-1,70)^{*}$ \\
\hline 1 & - & - & - & + & - & 4,7 & 3,6 & $1,31(1,04-1,59)^{*}$ & 2,2 & 1,4 & $1,62(1,28-1,96)^{*}$ \\
\hline 1 & - & - & - & - & + & 0,6 & 0,9 & $0,69(0,41-0,98)$ & 0,3 & 0,2 & $1,45(1,11-1,79)^{*}$ \\
\hline 2 & + & + & - & - & - & 4,9 & 3,2 & $1,53(1,26-1,79)^{*}$ & 5,5 & 4,6 & $1,19(0,89-1,49)$ \\
\hline 2 & + & - & + & - & - & 8,9 & 17,6 & $0,51(0,33-0,68)$ & 14,7 & 22,5 & $0,65(0,45-0,86)$ \\
\hline 2 & + & - & - & + & - & 11,4 & 9,7 & $1,18(0,95-1,41)$ & 8,6 & 9,6 & $0,90(0,62-1,17)$ \\
\hline 2 & + & - & - & - & + & 1,2 & 1,9 & $0,65(0,41-0,88)$ & 0,1 & 1,5 & $0,07(-0,24-0,38)$ \\
\hline 2 & - & + & + & - & - & 0,4 & 0,8 & $0,47(0,19-0,76)$ & 0,6 & 0,4 & $1,51(1,17-1,85)^{*}$ \\
\hline 2 & - & + & - & + & - & 0,4 & 0,5 & $0,86(0,58-1,14)$ & 0 & 0,2 & 0 \\
\hline 2 & - & + & - & - & + & 0,2 & 0,1 & $1,77(1,52-2,02)^{*}$ & 0 & 0,0 & 0 \\
\hline 2 & - & - & + & + & - & 1,2 & 2,6 & $0,47(0,19-0,75)$ & 0,6 & 0,8 & $0,73(0,39-1,07)$ \\
\hline 2 & - & - & + & - & + & 0 & 0,6 & 0 & 0 & 0,1 & 0 \\
\hline 2 & - & - & - & + & + & 2,0 & 0,3 & $5,87(5,60-6,15)^{*}$ & 0,1 & 0,1 & $1,86(1,54-1,18)^{*}$ \\
\hline 3 & + & + & + & - & - & 0,8 & 2,3 & $0,35(0,07-0,62)$ & 1,9 & 2,8 & $0,68(0,36-1,0)$ \\
\hline 3 & + & + & - & + & - & 3,3 & 1,3 & $2,62(2,33-2,90)^{*}$ & 2,1 & 1,2 & $1,75(1,45-2,09)^{*}$ \\
\hline 3 & + & + & - & - & + & 0,2 & 0,3 & $0,65(0,37-0,94)$ & 0,1 & 0,2 & $0,55(0,21-0,89)$ \\
\hline 3 & + & - & + & + & - & 1,0 & 6,9 & $0,14(-0,1-0,39)$ & 0,4 & 5,8 & $0,07(-023-0,37)$ \\
\hline 3 & + & - & + & - & + & 0,6 & 1,7 & $0,36(0,08-0,64)$ & 0 & 0,9 & 0 \\
\hline 3 & + & - & - & + & + & 1,6 & 0,7 & $2,43(2,14-2,71)^{*}$ & 1,3 & 0,4 & $3,43(3,09-3,77)^{*}$ \\
\hline 3 & - & + & + & + & - & 0 & 0,3 & 0 & 0,1 & 0,1 & $0,97(0,66-1,28)$ \\
\hline 3 & - & + & + & - & + & 0 & 0,1 & 0 & 0 & 0,0 & 0 \\
\hline 3 & - & + & - & + & + & 0 & 0 & 0 & 0 & 0,0 & 0 \\
\hline 3 & - & - & + & + & + & 0,4 & 0,2 & $1,64(1,38-1,91)^{*}$ & 0,1 & 0,2 & $0,43(0,09-0,77)$ \\
\hline 4 & + & + & + & + & - & 0,2 & 0,9 & $0,22(-0,06-0,51)$ & 0,4 & 0,7 & $0,55(0,21-0,89)$ \\
\hline 4 & + & + & + & - & + & 0 & 0,1 & 0 & 0 & 0,1 & 0 \\
\hline 4 & + & + & - & + & + & 0,2 & 0,1 & $1,67(1,41-1,92)^{*}$ & 0,3 & 0,0 & $6,34(6,02-6,65)^{*}$ \\
\hline 4 & + & - & + & + & + & 1,0 & 0,7 & $1,52(1,23-1,80)^{*}$ & 0,4 & 0,2 & $1,74(1,39-2,08)^{*}$ \\
\hline 4 & - & + & + & + & + & 0 & & 0 & 0 & 0,0 & 0 \\
\hline 5 & + & + & + & + & + & 0,2 & 0,1 & $2,33(2,10-2,56)^{*}$ & 0 & 0,0 & 0 \\
\hline
\end{tabular}

$\mathrm{n}=$ número de comportamentos; $(+)$ = comportamento presente; $(-)=$ comportamento ausente; $\mathrm{IA}=$ insuficientemente ativos; $\mathrm{CS}=$ comportamento sedentário excessivo; $\mathrm{FV}=$ frutas e verduras; $\mathrm{BA}=$ bebidas alcoólicas $\mathrm{T}=$ tabaco; $\mathrm{O} / \mathrm{E}=$ razão entre a prevalência observada e a prevalência esperada; IC = Intervalo de confiança; *presença de agregação.

\section{Discussão}

Os resultados deste estudo evidenciaram que os comportamentos de risco à saúde mais prevalentes foram os níveis insuficientes de atividade física e o consumo inadequado de frutas e verduras. A prevalência de três ou mais comportamentos agregados ficou abaixo de $10 \%$ e os adolescentes de famílias com renda inferior a dois salários mínimos apresentaram menores probabilidades de exposição a esse desfecho.

Em relação aos comportamentos de risco à saúde, a prevalência estimada de níveis insuficientes de ati- vidade física foi muito alta e significativamente maior para o sexo feminino. Estes dados preocupam pelo fato de que, independente do sexo, níveis insuficientes de atividade física na adolescência podem influenciar para uma vida adulta menos ativa ${ }^{15}$. A exposição, ainda na adolescência, a níveis insuficientes de atividade física indicam maiores chances de impactos negativos à saúde, bem como de risco de desenvolvimento precoce de doenças cardiovasculares e sobrepeso/obesidade ${ }^{2}$.

A prevalência encontrada para o uso de bebidas alcoólicas é preocupante, visto que o consumo nesta 
fase está associado a problemas na vida adulta, como doenças hepáticas e problemas comportamentais, além de aumentar as chances do uso excessivo ao longo da vida $^{16}$. Em relação ao uso de tabaco, apesar da baixa prevalência, o consumo na infância pode indicar continuidade na vida adulta. Estudo prévio ${ }^{17}$ indicou que a maioria dos adultos fumantes já era tabagista aos 18 anos, aumentando a chance de desenvolver doenças respiratórias e alguns tipos de cânceres.

A exposição à presença simultânea de três ou mais comportamentos de risco apresentou uma prevalência de 7,9\%. Estudos prévios observaram uma grande variação nas prevalências em adolescentes ${ }^{3,4,7,8}$. Uma possível explicação para a variação da prevalência entre adolescentes é o fato que, diferentemente dos adultos, estudos sugerem ${ }^{3,4}$ que neles alguns comportamentos tendem a se modificar ou não caso se apresentem combinados durante esta fase.

O presente estudo destacou a elevada prevalência e a agregação de níveis insuficientes de atividade física com os demais comportamentos de risco, em ambos os sexos. Isolada ou simultaneamente, estes comportamentos são evidenciados, em estudos prévios, como fatores de risco aos adolescentes e associados ao aumento de morbidade e mortalidade ${ }^{15,18,19}$.

Adolescentes de famílias com renda inferior a dois salários mínimos apresentaram menores probabilidades de exposição a três ou mais comportamentos de risco simultâneos. Estudos prévios realizados com adolescentes ${ }^{7-9}$ evidenciaram associações entre menor renda e presença de múltiplos comportamentos de risco. Apesar da menor probabilidade de exposição encontrada no presente estudo, baixo nível de escolaridade dos pais e renda familiar têm sido associadas ao desenvolvimento de padrões comportamentais específicos que aumentam os riscos precoces de morbidade e mortalidade ${ }^{1,2}$.

Como limitação do presente estudo, destaca-se o uso de questionário que, mesmo que validado e testado, poderia superestimar ou subestimar os valores de prevalência das variáveis relacionadas ao estilo de vida. Como pontos fortes, destacam-se a amostra representativa e que esse foi o primeiro levantamento epidemiológico sobre comportamentos de risco à saúde realizado no município investigado.

O estudo evidenciou a elevada prevalência de escolares insuficientemente ativos, do consumo inadequado de frutas e verduras, do aumento das chances de agregação entre consumo de bebidas alcoólicas e de tabaco para o sexo masculino. Para o sexo feminino, a elevada chance de agregação entre níveis insuficientes de atividade física, comportamento sedentário e consumo de bebidas alcoólicas e de tabaco. No entanto, escolares com renda familiar inferior à dois salários mínimos apresentaram menores chances de exposição à presença simultânea de três ou mais comportamentos.

Estes resultados trazem implicações importantes para subsidiar futuras intervenções, ao passo que os comportamentos de risco observados podem ser modificados, o que implicaria em estratégias de mudanças focadas nos múltiplos comportamentos agregados mais prevalentes e os respectivos fatores associados.

\section{Conflito de interesses}

Os autores declaram não haver conflito de interesses.

\section{Contribuição dos autores}

Munaro HLR, idealizou o projeto de pesquisa, coletou os dados, escreveu e revisou as análises estatísticas e o manuscrito. Silva DAS, auxiliou na concepção do projeto de pesquisa e todas as revisões e escrita do manuscrito. Lopes AS, auxiliou na concepção do projeto de pesquisa e todas as revisões e escrita do manuscrito. Todos os autores aprovaram a versão final do manuscrito.

\section{Agradecimentos}

Ao Núcleo Territorial de Educação da Bahia (NTE-22) por ter autorizado a execução do projeto de pesquisa "Comportamentos de risco à saúde de escolares de Jequié-Bahia”. A todos os gestores, professores e escolares por participarem da pesquisa. Ao DINTER entre a Universidade Federal de Santa Catarina e a Universidade Estadual de Santa Cruz, por proporcionar a realização do Doutorado Interinstitucional.

\section{Referências}

1. World Health Organization (WHO). Global recommendations on physical activity for health. Geneva, SW: World Health Organization, 2010.

2. Hallal PC, Andersen LB, Bull FC, Guthold R, Haskell W, Ekelund U, et al. Global physical activity levels: surveillance progress, pitfalls, and prospects. Lancet.2012;380(9838):247-57.

3. Lawlor DA, O'Callaghan MJ, Mamun AA, Williams GM, Bor W, Najman JM. Socioeconomic position, cognitive function, and clustering of cardiovascular risk factors in adolescence: findings from the Mater University Study of Pregnancy and its outcomes. Psyc Med. 2005;67:862-8.

4. Alamian A, Paradis G. Correlates of multiple chronic disease behavioral risk factors in Canadian children and adolescents. Am J Epidemiol. 2009;170(10):1279-89.

5. Plotnikoff RC, Karunamuni N, Spence JC, Storey K, Forbes L, Raine K, et al. Chronic disease-related lifestyle risk factors in a sample of Canadian adolescents. J Adolesc Health. 2009;44:606-9. 
6. Matos MG, Marques A, Calmeiro L, Loureiro N. Diferentes perfis comportamentais em adolescentes e associação à prática de atividade física. Psic Sau \& Doenças. 2014;15(2):495-509.

7. Dumith SC, Muniz LC, Tassitano RM, Hallal PC, Menezes AMB. Clustering of risk factors for chronic diseases among adolescents from Southern Brazil. Prev Med.2012;54(6):393-6.

8. Tassitano RM, Dumith SC, Chica DAG, Tenório MCM. Aggregation of the four main risk factors to noncommunicable diseases among adolescents. Rev Bras Epidemiol. 2014;17(2):465-78.

9. Leech RM, McNaughton SA, Timperio A. The clustering of diet, physical activity and sedentary behavior in children and adolescents: a review. Int J Behav Nutr Phys Act. 2014;11(4):1-9.

10. Instituto Brasileiro de Geografia e Estatística (IBGE). IBGE Cidades. [citado 2016 fev 20]. Disponível em: http://cidades.ibge.gov.br/xtras/perfil. php?lang=\&codmun=291800\&search=bahia $\mid$ jequie.

11. Luiz RR, Magnanini MMF. A lógica da determinação do tamanho da amostra em investigações epidemiológicas. Cad Saúde Col. 2000;8(2):9-28.

12. Silva KS, Lopes AS, Hoelfelmann LP, Cabral LGA, De Bem MFA, Barros MVG, et al. Health risk behaviors project (COMPAC) in youth of the Santa Catarina State, Brazil: ethics and methodological aspects. Braz J Kin Hum Per. 2013;15(1):1-15.

13. PeNSE. Pesquisa Nacional de Saúde do Escolar 2012. Rio de Janeiro: IBGE; 2013.
14. Reichenheim ME, Coutinho ES. Measures and models for causal inference in cross-sectional studies: arguments for the appropriateness of the prevalence odds ratio and related logistic regression. BMC Med Res Methodol. 2010;10(1):66.

15. Spring B, Moller AC, Coons MJ. Multiple health behaviors: overview and implications. J Public Health. 2012;34(S1):i3-i10.

16. Mccambridge J, Mcalaney J, Rowe R. Adult consequences of late adolescent alcohol consumption: a systematic review of cohort studies. PLOS Medi. 2011;8(2):1-13.

17. Currie C, Zanotti A, Morgan D, Currie D, Looze M, Roberts C. Social determinants of health and well-being among young people. Health Behavior in School-aged Children (HBSC) study: international report from the 2009/2010 survey W. R. O. F. Europe (Ed.). Health Policy for Children Adolesc. 2012;6.

18. Schuit AJ, van Loon AJ, Tijhuis M, Ocke M. Clustering of lifestyle risk factors in a general adult population. Prev Med. 2002;35(3):219-24.

19. Silva KS, Lopes AS, Vasques DG, Costa FF, Silva RCR. Clustering of risk factors for chronic noncommunicable diseases among adolescents: prevalence and associated factors. Rev Paul Ped. 2012;30(3):338-45.

Recebido: 02/06/2017 Aprovado: 23/07/2018

\section{Como citar este artigo:}

Munaro HLR, Silva DAS, Lopes AS. Agregação de comportamentos de risco à saúde de escolares da rede pública de Jequié, Babia. Rev Bras Ativ Fís Saúde. 2018;23:e0016. DOI: 10.12820/rbafs.23e0016 